\title{
Two-dimensional beams of dissipative antisolitons
}

\author{
A. Ankiewicz ${ }^{a}$, J. M. Soto-Crespo ${ }^{b}$, N. Devine ${ }^{a}$ and N. Akhmediev ${ }^{a}$ \\ ${ }^{a}$ Optical Sciences Group, Research School of Physical Sciences and Engineering, \\ The Australian National University, Canberra ACT 0200, Australia and \\ ${ }^{b}$ Instituto de Óptica, C.S.I.C., Serrano 121, 28006 Madrid, Spain
}

\begin{abstract}
By using a reduced model for dissipative optical soliton beams, we show that there are two disjoint sets of fixed points. These correspond to stationary solitons of the radial complex cubic-quintic Ginzburg Landau equation with concave and convex phase profiles, respectively. We confirm these results by numerical simulations which reveal soliton solutions of two different types: continuously self-focussing and continuously self-defocusing.
\end{abstract}

Keywords: Dissipative solitons, Ginzburg-Landau equation, method of moments.

\section{INTRODUCTION}

Optical beam propagation in nonlinear media has been studied in detail by a number of researchers [1-3]. In self-focusing conservative media, the main difference between the 1-dimensional (1-D) and 2-D cases is the problem of collapse [4-7]. In the simple model, 2-D beams are predicted to contract to an infinitely small spot with an infinite central field intensity. Various mathematical tools for the description of the collapse phenomenon have been suggested [1] and a variety of mechanisms to avoid it have been proposed [3].

The situation in dissipative media is quite different. There is no beam collapse because of the dissipation, which increases without limit as soon as the field increases to high values. The energy is created at some points of the beam and dissipated at others. Despite being a dynamical formation in this sense, the beam stays stationary: both the amplitude profile and the phase chirp across the beam stay fixed. In dissipative media, beam propagation can be studied using the complex Ginzburg-Landau equation (CGLE). Localized solutions to this equation are $2 \mathrm{D}$ beams in the three dimensional space with the longitudinal co-ordinate being the propagation direction. One complication is that there are surprisingly many types of localized solutions. Their classification is not an easy task.

In the present work, we find that there are at least two sets of solutions with a major qualitative difference: one set consists of "self-focusing" beams (solitons) and another set of "self-defocusing" beams (anti-solitons [8]). When smoothly changing the parameters of the medium, viz. the external pump, nonlinearity, spectral filtering, etc., the solutions also change smoothly in a given range, but abrupt changes occur at the bifurcation points. Our studies reveal the fact that one type of soliton cannot be transformed into the other - either smoothly or through a bifurcation. In fact, they are separated in parameter space by a region where no solitons exist. There is no way of continuously transforming a soliton from one region into a soliton of the other region.

The two dimensional beams under study belong to the class of "dissipative solitons" [9]. Thus, we can make certain conclusions based on our previous experience. Rosanov [10] was the first person to study cylindrically-symmetric solitons in laser cavities containing saturable nonlinear elements. As in his work, here we consider the simplest case of stationary 2-D solitons, where each one has a plain "bell-shaped" profile with a single maximum. This class of solutions can be reasonably well approximated with simple analytical trial functions, which allows us to predict the existence of two types of solutions using the method of moments or Lagrangian formalism [11]. The predictions are confirmed by using direct numerical simulations.

Complex Systems II, edited by Derek Abbott, Tomaso Aste, Murray Batchelor, Robert Dewar,

Tiziana Di Matteo, Tony Guttmann, Proc. of SPIE Vol. 6802, 68021C, (2008)

0277-786X/08/\$18 $\cdot$ doi: $10.1117 / 12.760937$

Proc. of SPIE Vol. 6802 68021C-1 


\section{BEAM PROPAGATION}

Our studies are based on an extended complex Ginzburg-Landau equation, that includes cubic and quintic nonlinear terms. This normalized propagation equation reads [12]:

$$
i \psi_{z}+\frac{D}{2} \nabla_{\perp}^{2} \psi+|\psi|^{2} \psi+\nu|\psi|^{4} \psi=i \delta \psi+i \epsilon|\psi|^{2} \psi+i \beta \nabla_{\perp}^{2} \psi+i \mu|\psi|^{4} \psi
$$

where $\psi(z, r)$ is the normalized envelope of the field,

$$
\nabla_{\perp}^{2} \psi=\frac{1}{r} \frac{\partial}{\partial r}\left(r \frac{\partial \psi}{\partial r}\right)
$$

is the transverse Laplacian for radially-symmetric beams, $z$ is the propagation distance and $r$ is the radial co-ordinate, $D$ is the diffraction coefficient that can be set to 1 without loss of generality, $\nu$ is the coefficient of the quintic nonlinearity, $\delta$ represents linear losses, $\epsilon$ is the nonlinear gain coefficient, $\beta$ stands for angular spectral filtering of the cavity, and $\mu$ characterizes the saturation of the nonlinear gain. This equation is applicable to wide aperture lasers $[13,14]$ and vertical external cavity semiconductor devices [15]. It can also be applied to multimode optical fibers made of erbium-doped glass, when the number of modes is very large.

Now, Eq.(1) does not have solutions in analytic form. The only way to obtain solutions is through numerical simulations. The latter requires a large amount of computer time. We are interested in the regions in the space of the parameters of Eq.(1) where stable soliton solutions exist. Before starting simulations, it would be helpful to have rough estimates where these solutions exist. Thus, we need approximate techniques that will allow us to guide our numerical simulations. The Lagrangian method [11] involves the reduction of the complete evolution problem with an infinite number of degrees of freedom to the evolution of a finite set of pulse characteristics. The problem of solving a partial differential equation is then reduced to solving a small set of ordinary differential equations (o.d.e.'s). For a localized solution with a single maximum, the main beam characteristics are the peak amplitude, the pulse width, and some phase chirp parameters. For any arbitrary localized field $\psi$, an important magnitude is its beam power $Q$ :

$$
Q=2 \pi \int_{0}^{\infty}|\psi|^{2} r d r
$$

In our low-dimensional approximation, we shall describe the pulse shape by using the beam power, $Q$, the width, $f$, and a chirp factor, $c$. These 3 non-zero quantities can give a good representation of the soliton amplitude and phase profiles.

Let $L$ be the integral of the Lagrangian density, $L_{d}$. Hence, we have

$$
L=L_{1}+\frac{D}{2} \mathcal{P}-\frac{1}{2} S_{4}-\frac{\nu}{3} S_{6}
$$

where, we have defined

$$
S_{n}=\int_{0}^{\infty}|\psi|^{n} r d r, \quad \mathcal{P}=\int_{0}^{\infty}\left|\frac{\partial \psi}{\partial r}\right|^{2} r d r . \quad \text { and } \quad L_{1}=-\frac{i}{2} \int_{0}^{\infty}\left(\psi^{*} \frac{\partial \psi}{\partial z}-\psi \frac{\partial \psi^{*}}{\partial z}\right) r d r .
$$

Then, $Q=2 \pi S_{2}$ is the total beam power. The rate of change of $Q$ is given by [12]

$$
Q_{z}=\frac{d Q}{d z}=4 \pi\left(\delta S_{2}-\beta P+\epsilon S_{4}+\mu S_{6}\right) .
$$

In the following, we shall use two trial functions that in combination with either the minimization of the Lagrangian or by using the method of moments allow us to approximate the real solution.

\section{QUARTIC-GAUSSIAN TRIAL FUNCTION}

In order to have more flexibility in approximating the beam shape, we used first a higher-order Gaussiantype function as a trial function, viz.

$$
\psi(r, z)=\frac{\sqrt{2}}{\pi^{3 / 4} f(z)} \exp \left[-\frac{r^{4}}{2 m^{2} f^{4}(z)}-\frac{r^{2}}{f^{2}(z)}\right] \sqrt{\frac{e^{-m^{2}} Q(z)}{m \operatorname{Erfc}(m)}} \exp \left[i\left(r^{2} c(z)-\theta(z)\right)\right],
$$


where $\operatorname{Erfc}(m)$ is the complementary error function. Here $\theta(z)$ is the axial phase evolution. For a stationary solution, $\theta^{\prime}(z)=\omega=$ const., so $\theta(z)=\omega z$. For pulsating solutions, $\omega=\omega(z)$ oscillates rather than being constant. The total beam power is thus $Q(z)$. Our studies on a related $1 \mathrm{D}$ problem [8] show that we can expect this type of function to give a good fit for the regions of existence of the solitons. So, in this case:

$$
\begin{gathered}
L_{1} \frac{2 \pi^{3 / 2}}{Q(z)}=m\left(\frac{e^{-m^{2}}}{\operatorname{Erfc}(m)}-m \sqrt{\pi}\right) f^{2}(z) c^{\prime}(z)-\sqrt{\pi} \theta^{\prime}(z) \\
S_{4}=\frac{\operatorname{Erfc}(\sqrt{2} m) Q^{2}(z)}{\sqrt{2} m \pi^{5 / 2} \operatorname{erfc}^{2}(m) f^{2}(z)} \\
S_{6}=\frac{2 \operatorname{Erfc}(\sqrt{3} m) Q^{3}(z)}{\sqrt{3} m^{2} \pi^{4} \operatorname{erfc}^{3}(m) f^{4}(z)} \\
\mathcal{P}=\frac{Q(z)}{\pi^{3 / 2} f^{2}(z)} \times\left[\frac{2 e^{-m^{2}}\left(m^{2} c^{2}(z) f^{4}(z)+1\right)}{m \operatorname{Erfc}(m)}-\sqrt{\pi}\left(2 m^{2} c^{2}(z) f^{4}(z)+1\right)\right]
\end{gathered}
$$

We have, from Ref.[11],

$$
\frac{d}{d z}\left(\frac{\partial L}{\partial p_{z}}\right)-\frac{\partial L}{\partial p}=2 \operatorname{Re}\left(\int_{0}^{\infty} K \frac{1}{i \psi^{*}} \frac{\partial \psi^{*}}{\partial p} r d r\right)
$$

for each parameter $p$, where $K$ indicates the dissipative terms of the CGLE, viz.,

$$
K=\delta|\psi|^{2}+\epsilon|\psi|^{4} \psi+\mu|\psi|^{6}+\beta \frac{\psi^{*}}{r} \frac{\partial}{\partial r}\left(r \psi_{r}\right) .
$$

We can then easily find $\frac{\partial L}{\partial p}$ and $\frac{\partial L}{\partial p_{z}}$ for each $p=Q, f, c, \theta, m$.

The first 3 multiplicative factors needed are purely imaginary:

$$
\begin{gathered}
\frac{1}{i \psi^{*}} \frac{\partial \psi^{*}}{\partial Q}=-\frac{i}{2 Q(z)}, \\
\frac{1}{i \psi^{*}} \frac{\partial \psi^{*}}{\partial f}=-\frac{i\left(\frac{2 r^{4}}{m^{2}}+2 f^{2}(z) r^{2}-f^{4}(z)\right)}{f^{5}(z)}, \\
\frac{1}{i \psi^{*}} \frac{\partial \psi^{*}}{\partial m}=\frac{i}{2}\left(-\frac{2 r^{4}}{m^{3} f^{4}(z)}+2 m+\frac{1}{m}-\frac{2 e^{-m^{2}}}{\sqrt{\pi} \operatorname{Erfc}(m)}\right) .
\end{gathered}
$$

The other 2 are real:

$$
\begin{gathered}
\frac{1}{i \psi^{*}} \frac{\partial \psi^{*}}{\partial c}=-r^{2}, \\
\frac{1}{i \psi^{*}} \frac{\partial \psi^{*}}{\partial \theta}=1 .
\end{gathered}
$$

For example,

$$
\frac{\partial L}{\partial \theta}=-\frac{Q(z)}{2 \pi}
$$


In fact, the $\theta$ equation recovers eqn.(3).

The trial function (4) can be improved in several ways to give a better fit for the exact solution. For example, we could use a different form for the phase term in (4). Namely, we could replace $c(z) r^{2}$ by the expression $b \log \left[\cosh \left(\frac{r}{f(z)}\right)\right]$. This form would reflect the fact that the phase tends to be roughly quadratic near the axis $(r=0)$ and turns into linear function when $r$ is larger. Near the center, $c \approx b / f^{2}$ and the sign of $b$ is the same as the sign of $c$. In reality, this modification does not lead to significant improvements.

Now we can either set the value of $m$, e.g. $m=$ const., and then find $Q, f, c$ or use the additional $m$ equation and solve for $Q, f, c$ and the "optimum" $m$. In practice, this latter idea leads to a more complicated dynamical system but does not give a significant improvement, so we usually study the evolution of the set of o.d.e's: $Q^{\prime}(z)=Q_{z}, f^{\prime}(z)=f_{z}, c^{\prime}(z)=c_{z}$ (and then we find $\theta^{\prime}(z)$ ), or just get the stationary solutions from the fixed points by solving the set $Q^{\prime}(z)=0, f^{\prime}(z)=0, c^{\prime}(z)=0$, and then use $\theta^{\prime}(z)=\omega$. The fixed points are designated $Q_{0}, f_{0}, c_{0}$. Various $m$ lead to different low-dimensional dynamical systems. Generally, they are complicated with coefficients which are given in terms of hypergeometric functions of $m$. Two relatively simple cases are presented below.

\section{A. The limit $m=1$}

We have used the trial function (4) with $m=1$ in the Lagrangian set of equations and obtained the following dynamical system for the variables $Q, f$ and $c$.

$$
\begin{aligned}
\frac{Q_{z}}{Q(z)} & =-2.55587 \beta c^{2}(z) f^{2}(z)+2 \delta+0.93407 \frac{\epsilon Q(z)}{f^{2}(z)}-6.55587 \frac{\beta}{f^{2}(z)}+0.547538 \frac{\mu Q^{2}(z)}{f^{4}(z)} \\
\frac{f_{z}}{f(z)} & =-0.98216 \beta c^{2}(z) f^{2}(z)+2 c(z)+2.147885 \frac{\beta}{f^{2}(z)}-0.194244 \frac{\epsilon Q(z)}{f^{2}(z)}-0.15927 \frac{\mu Q^{2}(z)}{f^{4}(z)} \\
c_{z} & =-2 c^{2}(z)-17.0404 \frac{\beta c(z)}{f^{2}(z)}-0.730924 \frac{Q(z)}{f^{4}(z)}+\frac{5.13004958}{f^{4}(z)}-0.57127423 \frac{\nu Q^{2}(z)}{f^{6}(z)} \\
\theta_{z} & =-5.44413 \beta c(z)-0.700555 \frac{Q(z)}{f^{2}(z)}+\frac{3.27793}{f^{2}(z)}-0.45628 \frac{\nu Q^{2}(z)}{f^{2}(z)}
\end{aligned}
$$

Clearly, the numerical coefficients in the above equations are approximate. If the solution is stationary, the right hand side of equation (18) is a constant $(=\omega)$. Then $\theta$ increases (decreases) linearly with $z$ depending on the sign of this constant.

\section{B. Gaussian limit}

The limit $m \rightarrow \infty$ in (4), corresponds to the Gaussian trial function:

$$
\psi=\frac{1}{f(z)} \sqrt{\frac{2 Q(z)}{\pi}} \exp \left[-\left(\frac{r}{f(z)}\right)^{2}\right] \exp \left[i\left(r^{2} c(z)-\theta(z)\right)\right] .
$$

The dynamical system in this case takes the simpler form:

$$
\begin{aligned}
\frac{Q_{z}}{Q(z)} & =-4 \beta c^{2}(z) f^{2}(z)+2 \delta+\left(\frac{2 \epsilon Q(z)}{\pi}-4 \beta\right) \frac{1}{f^{2}(z)}+\frac{8 \mu Q^{2}(z)}{3 \pi^{2} f^{4}(z)} \\
\frac{f_{z}}{f(z)} & =-2 \beta c^{2}(z) f^{2}(z)+2 c(z)+\left(2 \beta-\frac{\epsilon Q(z)}{2 \pi}\right) \frac{1}{f^{2}(z)}-\frac{8 \mu Q^{2}(z)}{9 \pi^{2} f^{4}(z)} \\
c_{z} & =-2 c^{2}(z)-8 \frac{\beta c(z)}{f^{2}(z)}+\left(2-\frac{Q(z)}{\pi}\right) \frac{1}{f^{4}(z)}-\frac{16 \nu Q^{2}(z)}{9 \pi^{2} f^{6}(z)} \\
\theta_{z} & =-4 \beta c(z)+\left(2-\frac{3 Q(z)}{2 \pi}\right) \frac{1}{f^{2}(z)}-\frac{20 \nu Q^{2}(z)}{9 \pi^{2} f^{4}(z)}
\end{aligned}
$$

Naturally, the only difference between this case and the case $m=1$ is the values of the coefficients in the right-hand-sides of the equations. 


\section{SUPER-GAUSSIAN TRIAL FUNCTION}

Originally, a Gaussian trial function was used to study the self-focusing of light beams in a conservative saturable medium [16]. Later, a "super-Gaussian" function was used [17]. These works studied localized solutions of the nonlinear Schrödinger equation with an additional term to include nonlinear saturable refraction, but did not consider any loss/gain terms. We now use this type of function for the dissipative CGLE. To be specific, we use:

$$
\psi=\frac{1}{f(z)} \sqrt{\frac{4^{\frac{1}{k}} Q(z)}{\pi \Gamma\left(\frac{k+2}{k}\right)}} \exp \left[-\left(\frac{r}{f(z)}\right)^{k}\right] \exp \left[i\left(r^{2} c(z)-\theta(z)\right)\right],
$$

so that the total beam power is again $Q(z)$. We find the dynamical system for general $k>1$ :

$$
\begin{gathered}
\frac{Q_{z}}{Q(z)}=2 \delta-\frac{4^{1+\frac{1}{k}} \beta c^{2}(z) f^{2}(z) \Gamma\left(\frac{1}{2}+\frac{2}{k}\right)}{\sqrt{\pi}}+\frac{2^{\frac{k+4}{k}} 9^{-1 / k} \mu Q^{2}(z)}{\pi^{2} \Gamma^{2}\left(\frac{k+2}{k}\right) f^{4}(z)}-\frac{\left(4^{\frac{1}{k}} \beta k \pi-2 \epsilon Q(z)\right)}{\pi \Gamma\left(\frac{k+2}{k}\right) f^{2}(z)} . \\
\frac{f_{z}}{f(z)}=2 c(z)+2^{\frac{k-2}{k}} \beta c^{2}(z) f^{2}(z)\left(\frac{16^{\frac{1}{k}} \Gamma\left(\frac{1}{2}+\frac{2}{k}\right)}{\sqrt{\pi}}-\frac{2 \Gamma\left(\frac{6}{k}\right)}{\Gamma\left(\frac{4}{k}\right)}\right)-\frac{\left(\frac{16}{81}\right)^{\frac{1}{k}}\left(-1+9^{\frac{1}{k}}\right) \mu Q^{2}(z)}{\pi^{2} f^{4}(z) \Gamma^{2}\left(\frac{k+2}{k}\right)} \\
+\frac{2^{\left(\frac{2}{k}-1\right)}\left(\Gamma\left(\frac{1}{2}+\frac{2}{k}\right)\left(16^{\frac{1}{k}} \beta k \pi-2\left(-1+4^{\frac{1}{k}}\right) \epsilon Q(z)\right)-2 \pi^{3 / 2} \beta(k-2) \Gamma\left(\frac{k+2}{k}\right)\right)}{\pi^{3 / 2} f^{2}(z) \Gamma\left(\frac{k+4}{k}\right)} .
\end{gathered}
$$

Now we find $c_{z}$ :

$$
c_{z}=-2 c^{2}(z)-\frac{g}{f^{2}(z) h}+\frac{(a-b)}{f^{4}(z) d}
$$

where

$$
\begin{gathered}
g=4^{\frac{k-1}{k}} \beta k \sqrt{\pi} c(z), \quad h=\Gamma\left(\frac{1}{2}+\frac{2}{k}\right), \\
a=4^{\frac{1}{k}} 3 \pi\left(4^{\frac{1}{k}} k \pi-2 Q(z)\right), \quad b=\frac{8^{\frac{k+2}{k}} 9^{-1 / k} \nu Q^{2}(z)}{f^{2}(z) \Gamma\left(\frac{k+2}{k}\right)} \quad \text { and } \quad d=6 \pi^{2} \Gamma\left(\frac{k+4}{k}\right) .
\end{gathered}
$$

The equation for $\theta$ takes the form:

$$
\theta_{z}=\frac{k\left(-24 \beta \pi^{2} c(z) \Gamma^{2}\left(\frac{2}{k}\right) f^{4}(z)+3 \pi \Gamma\left(\frac{2}{k}\right)\left(4^{\frac{1}{k}} k \pi-3 Q(z)\right) f^{2}(z)-5\left(\frac{16}{9}\right)^{\frac{1}{k}} k \nu Q^{2}(z)\right)}{12 \pi^{2} \Gamma^{2}\left(\frac{2}{k}\right) f^{4}(z)} .
$$

Then the Gaussian limit function (19) and equations are found by taking $k=2$. This reproduces eqns.(20) to (23). We can check the NLSE case by setting the parameters responsible for gain or loss in the equation to zero. In that case, we find that the power is

$$
Q=2^{\frac{2}{k}-1} k \pi
$$

and this has its minimum (and optimum) value when $k=\log 4=2 \log 2 \approx 1.38629$. Then $Q / \pi=e \log 2=$ 1.88417, which is close to the exact value of 1.86226. This agrees with the values found in Ref. [18]. In fact, we can use this value of $k$ for finding solutions of the o.d.e's for arbitrary parameter values. Again, we obtained regions where the chirp is negative, and regions where it is positive. Both trial functions allow us to find regions of stable solitons with reasonable accuracy. 


\section{RESULTS OF THE LOW-DIMENSIONAL APPROXIMATIONS}

We are interested in stationary localized solutions (see fig.1a). Stationary solutions of eqns.(15) to (18) are given by the soliton parameters which are $z$ independent, i.e. $Q(z)=Q_{0}, f(z)=f_{0}$ and $c(z)=c_{0}$. When we have these, we can use eqn. (18) to find $\theta_{z}=\omega$. The latter case corresponds to a fixed point (FP) of the dynamical system (18). Fixed points of this three-variable dynamical system, together with the trial function (4), approximate the stationary solitons of the CGLE. Standard linearization techniques can be used to find the stability of these FPs.
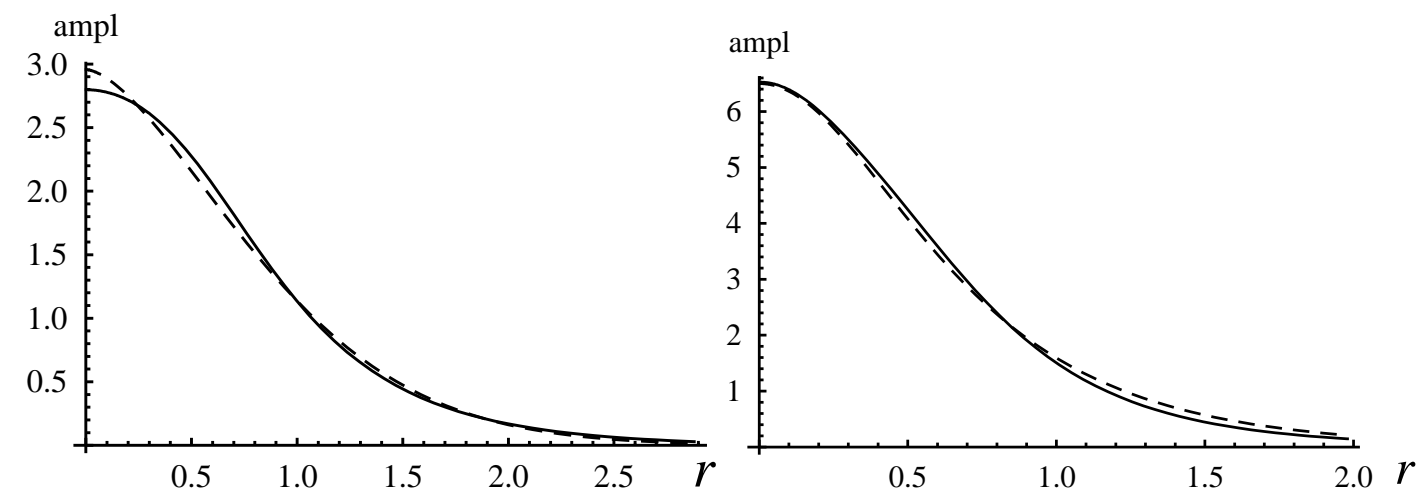

FIG. 1: (a) Soliton amplitude profile with negative chirp $(c<0)$. Exact soliton amplitude profile (solid line) and super-Gaussian approximation (24) with $M=1.6$ (dashed line). Here, $\delta=-0.1, \nu=-0.08, \mu=-0.03, \epsilon=0.22$, $\beta=0.08$. The values for power $Q=13.8$, width $f=1.03$, chirp $c=-0.058$ and propagation constant $\omega=-1.2$ predicted with the low-dimensional approximation fit the exact results fairly well. (b) Beam profile when the chirp is positive $(c>0)$. Exact profile is shown by the solid line while the approximation is shown by the dashed line. Here, $\delta=-0.045, \nu=-0.08, \mu=-0.05, \epsilon=3, \beta=2.5$, and power $Q \approx 42.7$

An investigation of the parameter space, using as trial function the eq.(4), shows up a region of positively chirped solitons. For the soliton whose amplitude profile is given in fig.1b, the phase can be fitted well by the function $b \log [\cosh (r / f(z))]$ with $b=1.2$ and width $f(z)=0.48$. So the magnitude of the positive chirp is high compared with that of the negative chirp case of fig.1a. Using parameters $\epsilon=3, \nu=-0.08 \mu=-0.03$ and $\delta=-0.1$, we can vary $\beta$ and observe various behaviors. In particular, we can get fixed points (FPs) with $c<0$ and FPs with high power $Q$ and $c>0$.

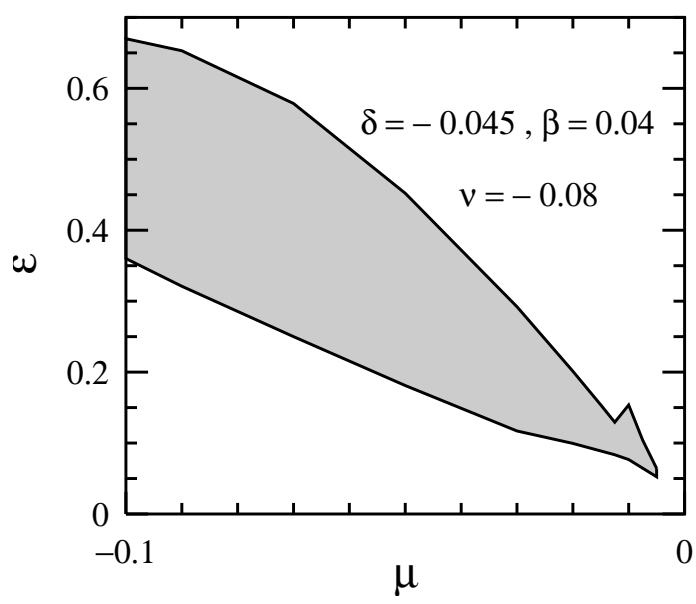

FIG. 2: Region of existence of solitons with negative chirp $\left(c_{0}<0\right)$ in the $(\mu, \epsilon$,$) plane found using the trial function$ (4). Stable solitons exist in the gray region.

By solving the dynamical system (15)-(18) for various values of the equation parameters, we can determine regions of stable and unstable fixed points in the space of the parameters of the CGLE. In order to keep 
the presentation of the results reasonably simple, we fix the value of three parameters and change the other two. In this way we find two-dimensional slices in the five-dimensional space of the equation parameters. An example of such plot is given in Fig.2 for the case $c<0$. We have found two separate regions of stable fixed points. A point from one region cannot be transformed into a point from the other region with a continuous change of parameters. Thus, it appears that these two regions correspond to two different types of solitons of the CGLE. One of them has high power, $Q$, while the other one has low power. Within the low-dimensional approximation (15)-(18), these FPs are stable in both regions.

For example, let us take $\epsilon=3, \nu=-0.08, \mu=-0.05, \delta=-0.045$. In this case, for $1.3<\beta$, we get stationary solitons with the chirp, $c$ and frequency offset, $\omega$ being positive. When $c$ changes sign, so does the phase front and direction of energy flux across the beam. The energy may be generated in the center and flow outwards (self-defocusing), or be generated in the outer layers and flow to the central region where it is dissipated. In the latter case, the beam is of self-focusing type.

In contrast to one-dimensional case [8], here we were not able to plot regions of existence of two types of solitons on the same two-dimensional plane. The two regions are separated completely in the space of the equation parameters. Nevertheless, we can see clearly that solitons of the second type require much higher $\epsilon$ values for their existence. Their powers $Q$ are also usually higher.

\section{DIRECT SIMULATIONS}

Direct simulations of the CGLE (1) confirm our predictions made using the simple model. In this case, we do not assume radial symmetry of the beams and therefore the Laplacian is now:

$$
\nabla_{\perp}^{2}=\frac{\partial^{2}}{\partial x^{2}}+\frac{\partial^{2}}{\partial y^{2}}
$$

We have numerically solved Eq.(1) in a Cartesian grid using a split-step Fourier method. Thus, the secondorder derivative terms in $x$ and $y$ are solved in Fourier space. All other linear and nonlinear terms in the equation are solved in real space using a fourth-order Runge-Kutta method. Most of the simulations presented in the paper were carried out using a numerical grid of $512 \times 512$ points in $(x, y)$. We used various values of step sizes along the $x, y$ and $z$ variables to check that the results do not depend on the mesh intervals, thus avoiding any numerical artifacts. In this way, we were able to find regions where stable stationary solutions exist and we determined the exact field amplitude and phase profiles of the solutions in these regions. The phase profiles clearly show that the chirps can be of opposite sign. Due to this difference, the energy flows from the center to the outside of the soliton in one case while it flows inwards in the other.
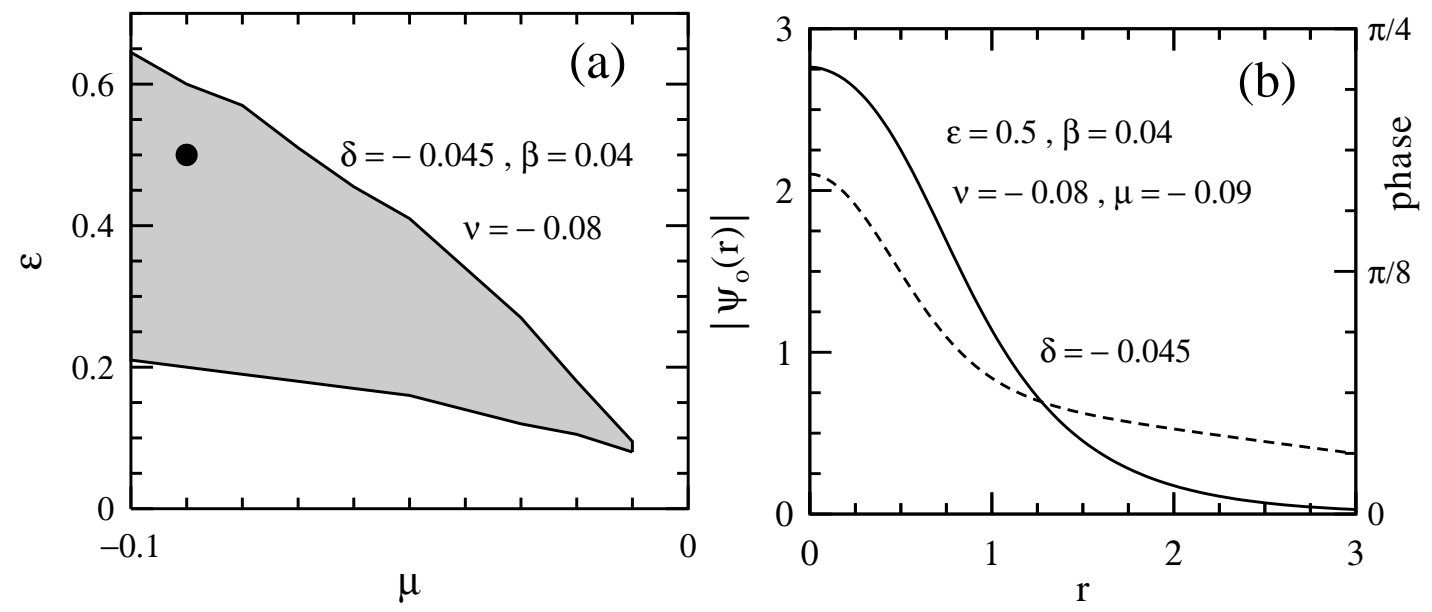

FIG. 3: (a) Region of existence of solitons with negative chirp $\left(c_{0}<0\right)$ in the $(\mu, \epsilon$, ) plane (direct simulation results). Stable solitons exist in the gray region. (b) Amplitude (solid line) and phase (dashed line) profiles of the soliton at the point shown by the thick black point at (a). The values of the rest of parameters are $\beta=.04, \nu=-0.08$, $\delta=-.045$. Above the gray region in (a), solitons lose the radial symmetry and are transformed into rotating double soliton complexes. 
The results of direct numerical simulations are shown in Figs. 3 and 4. Figure 3 shows the results for solitons (negative chirps) while Fig.4 is for antisolitons (positive chirps). Positive chirp regions are found at higher values of $\beta$ and $\epsilon$. A typical soliton solution inside the region of existence of stable solutions (gray area in Fig.3a) is shown in Fig.3b. These solutions have radial symmetry. The solid line represents the amplitude profile whereas the dashed line its phase profile. Similar plots for antisolitons are shown in Figs.4a and 4b. As stated above, the amplitude profiles are similar in both cases but the curvature of the phase profile has opposite sign to that of the solutions in Fig. 3. As a rule, the phase change is stronger for antisolitons. This is seen clearly from Fig.4b. Within the interval shown, the phase changes by more than $2 \pi$, while in the case of Fig.3b, the phase changes only by $\pi / 8$. The reduced model roughly predicts the amplitudes, chirps and energies of these beams.
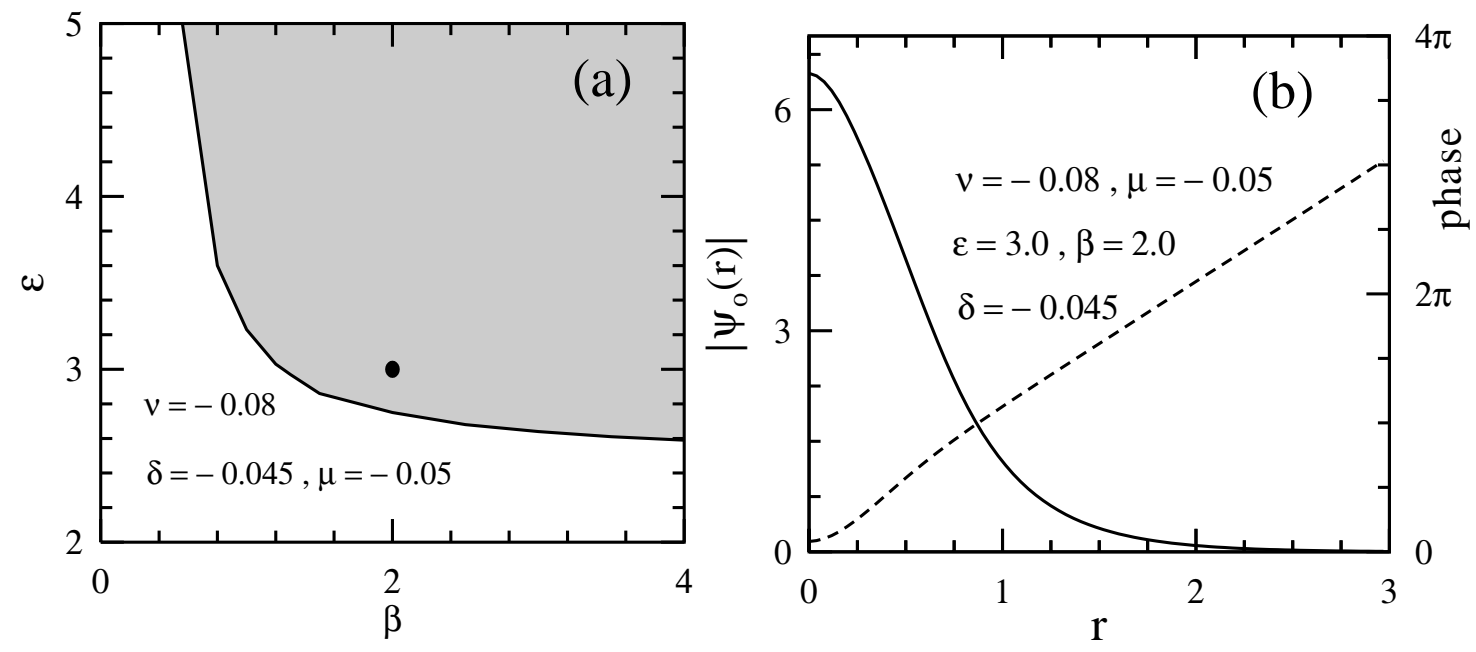

FIG. 4: (a) Region of existence of anti-solitons (self-defocusing type of solitons) in $(\beta, \epsilon)$ plane. The other parameters are $\nu=-0.08, \mu=-0.05, \delta=-0.045$. (b) Amplitude (solid line) and phase (dashed line) profiles of the anti-soliton with $\epsilon=3, \beta=2$ indicated in (a) by the solid black dot.

\section{RADIAL ENERGY FLOW IN THE BEAM}

We can use the reduced model to conveniently relate the chirp $c_{0}$ to the energy generation and flux across the beam. These values satisfy the continuity equation for any given system. For stationary solutions, the radial density of energy generation, $\hat{P}(r)$ is related to the radial flux $\hat{\jmath}(r)$ through the following equation:

$$
\hat{P}(r)=\frac{1}{r} \frac{\partial}{\partial r}(r \hat{\mathrm{j}})
$$

where

$$
\hat{\jmath}=\frac{i D}{2}\left(\psi \psi_{r}^{*}-\psi_{r} \psi^{*}\right)
$$

and

$$
\hat{P}(r)=2 \delta|\psi|^{2}+2 \epsilon|\psi|^{4}+2 \mu|\psi|^{6}+\beta\left(|\psi|_{r r}^{2}-2\left|\psi_{r}\right|^{2}+\frac{1}{r} \frac{\partial}{\partial r}|\psi|^{2}\right) .
$$

Using the trial function (4) for arbitrary $m$, we find the flux to be

$$
\hat{\jmath}=\frac{4 R c_{0} Q_{0}}{m \pi^{3 / 2} \operatorname{Erfc}(m) f_{0}} \exp \left[-\frac{\left(m^{2}+R^{2}\right)^{2}}{m^{2}}\right],
$$


where $R=r / f_{0}$. Then the energy generation $\hat{P}$ is given by

$$
\hat{P}(R)=\frac{8\left(m^{2}\left(1-2 R^{2}\right)-2 R^{4}\right) c_{0} Q_{0}}{m^{3} \pi^{3 / 2} \operatorname{erfc}(m) f_{0}^{2}} \exp \left[-\frac{\left(m^{2}+R^{2}\right)^{2}}{m^{2}}\right] .
$$

Normalized values of $P(R)=\hat{P} \frac{f_{0}^{2}}{c_{0} Q_{0}}$ and $j(R)=\hat{\jmath} \frac{f_{0}^{2}}{c_{0} Q_{0}}$ are plotted in Fig.5. These values do not depend on the chirp. $P(R)$ is positive at the center of the beam while $j(R)$ is positive in the whole interval.

We have the condition that the total energy generation is zero:

$$
\int_{0}^{\infty} R P(R) d R=0
$$

This condition has to be satisfied for stationary solutions, and is clearly valid here for all $m$. As we can see from (32) and (33), the values $\hat{\jmath}$ and $\hat{P}$ are directly proportional to $c_{0}$.

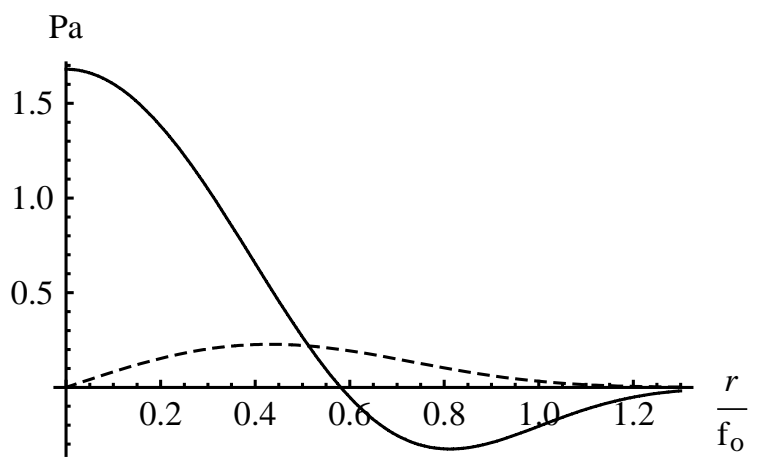

FIG. 5: Normalized energy generation $P$ as a function of the normalized radius $R=r / f_{0}$. We used $b \log \left[\cosh \left(\frac{r}{f(z)}\right)\right]$ as the phase term in eqn.(4) and $m=1$. Dashed line represents normalized radial flux $j\left(r / f_{0}\right)$.

If $c_{0}<0$, energy is generated in the outer parts of the beam where $\hat{P}>0$, and flows to the central area where it is dissipated (see Fig.6a). In a conservative medium, this process would correspond to the beam self-focusing. If $c_{0}>0$, then the process is the opposite: the energy is generated near to the central axis where $\hat{P}>0$, and it is dissipated in the outer parts of the beam where $\hat{P}<0$ (see Fig.6b). Again, in a conservative medium, this would correspond to the self-defocusing of the beam. Clearly, the beam could not be stationary when either self-focusing or self-defocusing occurs. However, in dissipative media, this effect, involving an internal radial flux of energy in the beam, produces the dynamical equilibrium within the stationary solution.

If we replace $c(z) r^{2}$ by the expression $b \log \left[\cosh \left(\frac{r}{f(z)}\right)\right]$ in eqn.(4), we obtain, for the flux:

$$
\hat{\mathrm{j}}=\frac{2 \tanh (R) b Q_{0}}{m \pi^{3 / 2} \operatorname{Erfc}(m) f_{0}^{3}} \exp \left[-\frac{\left(m^{2}+R^{2}\right)^{2}}{m^{2}}\right]
$$

For the energy generation we obtain the following expression:

$$
P=\frac{2 m^{2} R \operatorname{sech}^{2}(R)+2\left[m^{2}\left(1-4 R^{2}\right)-4 R^{4}\right] \tanh (R) b Q_{0}}{m^{3} \pi^{3 / 2} R \operatorname{Erfc}(m) f_{0}^{4}} \exp \left[-\frac{\left(m^{2}+R^{2}\right)^{2}}{m^{2}}\right] .
$$

We plot the normalized forms of $P(t)=\frac{\hat{P} f_{0}^{4}}{b Q_{0}}$ and $j(t)=\frac{\hat{\jmath} f_{0}^{3}}{b Q_{0}}$ in Fig.5b. The direction of energy flow is now determined by the sign of the chirp parameter $b$ which is the same as the sign of $c$.

The different signs of chirp for the two types of solitons mean that, in one case, the on-axis phase is retarded relative to the outer part (negative $c$ or $b$ ), while in the other case it is advanced with respect to the outer part (positive $c$ or $b$ ). In the first case, energy is generated in the outer parts of the soliton and 
dissipated near the center of the beam, while in the second case the energy generation is positive near the axis of the soliton and negative in the wings.

To be specific, we can call beams of self-focusing type "dissipative solitons" (negative $c$ or $b$ ) and designate beams of self-defocusing type as "dissipative antisolitons" (positive $c$ or $b$ ), in analogy with the 1D case [8]. Generally, antisolitons tend to have high positive chirp and higher power $Q$ than negatively chirped solitons.
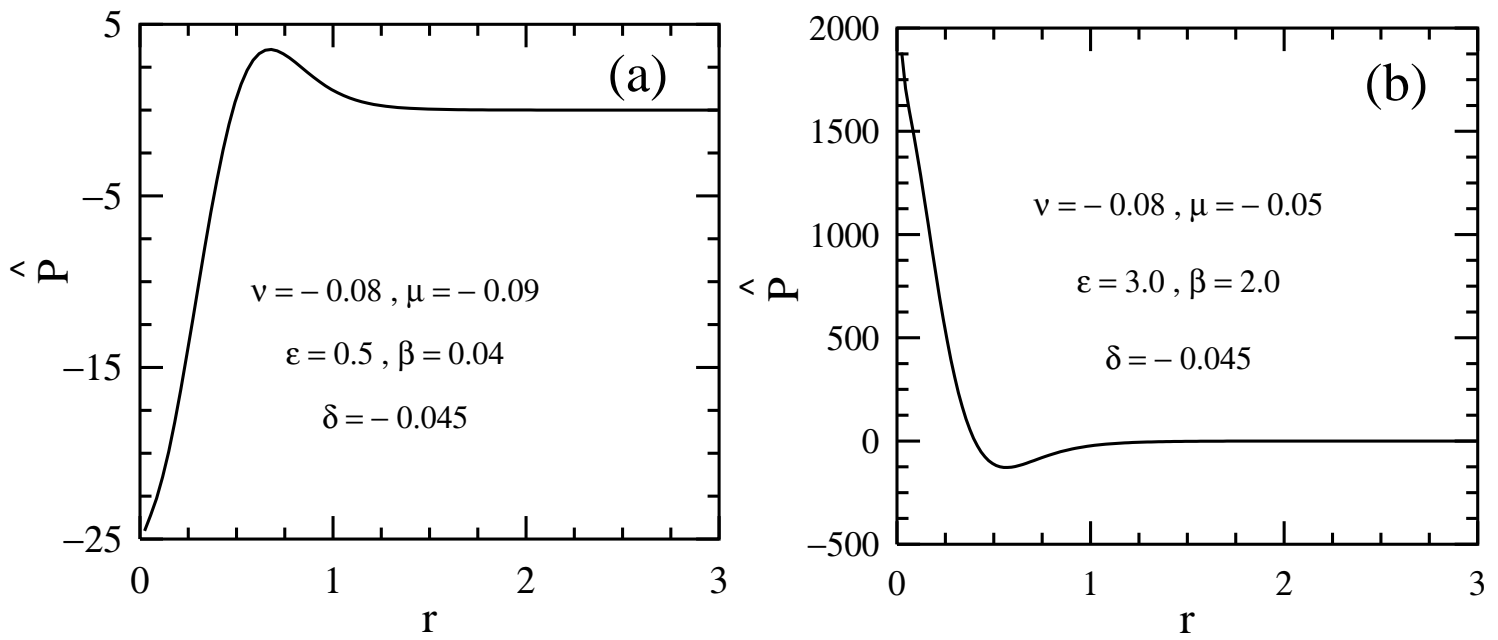

FIG. 6: Energy generation curves $\hat{P}$ as a function of the radius $r$ for (a) solitons and (b) antisolitons. The data are for the numerical soliton solutions presented in Figs.3b and $4 \mathrm{~b}$ respectively.

\section{CONCLUSION}

In conclusion, we have shown that there are two types of stable fixed points in the reduced system representing dissipative solitons of the radially-symmetric CGLE. The parameter regions of these solitons are quite separate. We have shown that these two types of solitons have opposite signs of the chirp parameter which governs the direction of energy flow inside the soliton beam. The predictions of the reduced approach have been confirmed by direct simulation of the CGLE.

This research was supported under Australian Research Council's Discovery Projects funding scheme (project number DP0663216). The work of J.M.S.C. was supported by the M.E.y C. under contract FIS200603376 .

\section{REFERENCES}

[1] V. Zakharov, V. Synakh, The nature of self-focusing singularity, Zh. Eksp. Teor. Fiz. 68 (1975), 940-947; Sov. Phys. JETP 41 (1975), 465-468.

[2] N. Akhmediev, A. Ankiewicz and J. M. Soto-Crespo, Does the nonlinear Schroedinger equation correctly describe beam propagation? Optics Letters, 18, 411 (1993).

[3] G. Fibich, Small beam nonparaxiality arrests self-focusing of optical beams, Physical Review Letters, 76, 43564359, (1996).

[4] M. D. Feit and J. A. Fleck, J. Opt. Soc. Am. B 5, 633 (1988).

[5] J. T. Manassah and B. Gross, Opt. Lett. 17, 976 (1992).

[6] V.E. Zakharov, Collaps of Langmuir waves, Zh. Eksp. Theor. Fiz., vol. 62,. p. 1747-1759 (1972) (in Russian); Sov. Phys. JETP 35, 908-914 (1972).

[7] L. Berge, Wave collapse in physics: principles and applications to light and plasma waves, Physics Reports, 303, 262 (1998).

[8] A. Ankiewicz, N. Devine, N. Akhmediev and J.M. Soto-Crespo, "Dissipative solitons and antisolitons", Physics Letters A, 370, 454 (2007). 
[9] N. Akhmediev and A. Ankiewicz, Dissipative Solitons in the Complex Ginzburg-Landau and Swift-Hohenberg Equations, In: Dissipative solitons, edited by N. Akhmediev and A. Ankiewicz, Springer-Verlag, Berlin (2005).

[10] N. N. Rosanov, Solitons in laser systems with saturable absorption, in: Dissipative solitons, edited by N. Akhmediev and A. Ankiewicz, Springer-Verlag, Berlin (2005), p.101.

[11] A. Ankiewicz, N.Devine, N. Akhmediev, Jnl. Opt fib.tech., 13, 91-97 (2007).

[12] See e.g. N. Akhmediev and A.Ankiewicz, Solitons: nonlinear pulses and beams, Chapman \& Hall, London, 1997, chap.11; G. P. Agrawal, Nonlinear Fiber Optics, (Academic Press, London, 1989).

[13] R. Meucciyx, A. Labatey, M. Ciofiniy and Peng-Ye Wangz, Bifurcations of hexagonal patterns stabilized and selected with spatial perturbations in a wide-aperture laser, Quantum Semiclass. Opt. 10, 803-808 (1998).

[14] S. V. Fedorov, N. N. Rosanov, A. N. Shatsev, N. A. Veretenov and A. G. Vladimirov, Topologically Multicharged and Multihumped Rotating Solitons in Wide-Aperture Lasers With a Saturable Absorber, IEEE J. of Quantum Electronics, 39, No. 2, 197 (2003).

[15] J.-F. Mercier and J. V. Moloney, Derivation of semiconductor laser mean-field and Swift-Hohenberg equations, Phys. Rev. E 66, 036221 (2002)

[16] D.Anderson and M.Bonnedal, Phys.Fluids, 22, 105 (1979).

[17] M.Karlsson, Phys.Rev. A, 46, 2726 (1992).

[18] A. Ankiewicz and N. Akhmediev, IEE J:optoelectronics, 150, 519-526 (2003). Note that the normalization in $\mathrm{r}$ differs from this work by $\sqrt{2}$ and so energy definitions differ by a factor of 2 .

[19] M. N. Zhuravlev and N. V. Ostrovskaya, J. Exper. Theor. Phys. 99, 427 (2004) [ Zh. Eksp. Teor. Fiz. 126, 483 (2004), in Russian].

[20] E. N. Tsoy and N. Akhmediev, Phys. Lett. A 343, 417-422 (2005).

[21] E. N. Tsoy, A. Ankiewicz and N. Akhmediev, Phys. Rev. E 73, 036621 (2006).

[22] J. M. Soto-Crespo, N. Akhmediev and A. Ankiewicz, 'Pulsating, Creeping, and Erupting Solitons in Dissipative Systems', Physical Review Letters, vol. 85, 2937-2940, (2000).

[23] N. Akhmediev, J. M. Soto-Crespo, and G. Town, Pulsating solitons, chaotic solitons, period doubling, and pulse coexistence in mode-locked lasers: CGLE approach, Phys. Rev. E, 63056602 (2001).

[24] A.Fernandez et al., Opt.Lett., 29 1366-8 (2004).

[25] N. Akhmediev and J. M. Soto-Crespo, "Exploding solitons and Shil'nikov's Theorem", Phys. Lett. A, 317, 287 $-292(2003)$ 